\title{
Cyclin-dependent kinase (CDK) 4/6 inhibitors in oncology
}

\author{
Evangelos Karamitrousis ${ }^{1}$, Michalis Liontos ${ }^{2}$, Nikolaos Tsoukalas ${ }^{3 *}$ \\ ${ }^{1}$ Department of Medical Oncology, Democritus University of Thrace, \\ Alexandroupolis, Greece \\ 2Department of Clinical Therapeutics, Alexandra Hospital, Athens, Greece \\ ${ }^{3}$ Department of Oncology, 401 General Military Hospital, Athens, Greece
}

The new issue of our journal is a fact and contains articles of great interest. In this issue, special reference is made to the system of cyclin-dependent kinases (CDK). Two articles are published: one about the pharmacological profile and management of CDK4/6 inhibitors in advanced breast cancer (1) and the other about the role of cyclin D1-DJ1 in prostate cancer and benign prostatic hyperplasia (2).

CDK4/6 inhibitors, palbociclib, ribociclib, and abemaciclib, in combination with hormone therapy have radically changed the management of patients with advanced hormone receptor-positive, HER2-negative breast cancer. These drugs prevent $\mathrm{G} 1$ to $\mathrm{S}$ cell cycle transition by inhibiting CDK 4 and 6 and have proved substantial efficacy as first-line therapy and subsequent lines of therapy in advanced/metastatic breast cancer.

\section{References}

[1] Tzelepi C. Vasiliki, Gogadis T. Aristeidis, Adamidis K. Christos et al, The Pharmacological Profile of Cyclin-dependent Kinase (CDK) 4/6 Inhibitors: Clinical Management of Toxicity and Drug Interactions Related to CDK 4/6 Inhibitor-based Treatment in Advanced/Metastatic Breast Cancer. Forum ClinOncol 2019 10(2): 2-14.

[2] Amrallah A. Mohammed, Hanna M. Ibrahim, Hanna A. Atwa et al, Impact of cyclin D1 and DJ-1 on diagnosis, clinico-pathological features and outcome in prostate cancer and benign prostatic hyperplasia. Forum ClinOncol 2019 10(2): 15-25.
Their pharmacological profile is favorable as they are generally well tolerated, and the common side effects include nausea, fatigue, diarrhea, and myelotoxicity.

Ongoing questions regarding predictive biomarkers for the use of these agents, their safety and efficacy in other breast cancer populations as well as other neoplasms are under investigation. Articles included in the current FCO issue attempt to provide further insight into these scientific questions. Large randomized clinical trials are currently underway that will form the therapeutic landscape with these drugs in the near future.

Under this perspective, the editorial team of Forum of Clinical Oncology is excited toward the current issue and we hope this will serve as the stepping stone for the continuous growth of the journal. 